Perfor mance measures of al cohol-i nduced i mpai r ment: Towar ds a pract i cal i gni ti on- i nt erl ock syst em for not or vehi cles

\begin{tabular}{|l|l|}
\hline 著者 & $\begin{array}{l}\text { Nat sumur a Kent a, Yanakoshi Takehi ro, I da } \\
\text { Takayuki }\end{array}$ \\
\hline $\begin{array}{l}\text { j our nal or } \\
\text { publ i cat i on t i t l e }\end{array}$ & Per cept ual and Not or Ski I I s \\
\hline vol une & 109 \\
\hline number & 3 \\
\hline page r ange & $841-850$ \\
\hline year & 2009-12-01 \\
\hline URL & ht t p: //hdl . handl e. net /2297/23906 \\
\hline
\end{tabular}




\section{PERFORMANCE MEASURES OF ALCOHOL-INDUCED IMPAIRMENT: TOWARDS A PRACTICAL IGNITION- INTERLOCK SYSTEM FOR MOTOR VEHICLES ${ }^{1,2}$}

\section{KENTA MATSUMURA}

National Center of Neurology and Psychiatry
TAKEHIRO YAMAKOSHI AND TAKAYUKI IDA

Kanazawa University

Summary.-Performance-based alcohol screening devices may help reduce road traffic accidents, but there is a shortage of easy-to-use performance tests available. To address this issue, four recently developed rapid, computerized, easily implementable performance tests, Spiral for iPhone and Spiral for Mac (psychomotor tests), and the Modified Mental Rotation and Catch the Rabbit tests (cognitive tests), were assessed, testing participants at predrink baseline and then during three progressive amounts of alcohol intake. Analyses showed all tests were performed statistically significantly less accurately at $0.11 \%$ blood alcohol concentrations (BACs) than at $0.00 \%$ BAC, as were all tests except Spiral for iPhone at $0.06 \%$ BAC. These results indicate the suitability of all of these tests for measuring alcoholinduced impairment, and some potential for use as a practical performance-based alcohol screening device.

Driving after drinking alcohol is associated with motor vehicle accidents, especially those with a fatal outcome. According to recent Japanese motor vehicle accident statistics, ${ }^{3}$ the death rate in such accidents is about 30 times as high as that in accidents without consumption of alcohol. It is well known and generally accepted that the dangers of driving after consuming alcohol result from alcohol-induced impairment (Mitchell, 1985; Moskowitz \& Burns, 1990). Driving depends upon accurate use of highly complex mental and motor skills, and alcohol has been shown to impair a wide range of these psychomotor and cognitive skills which are prerequisites for performing driving tasks adequately. For example, alcohol impairs the performance of tests concerned with vigilance (Gustafson, 1986; Hindmarch, Kerr, \& Sherwood, 1991), sustained or divided attention (Koelega, 1995), eye movements (Stapleton, Guthrie, \& Linnoila, 1986; King \& Byars, 2004), spatial information processing (Matthews, Best, White, Vandergriff, \& Simson, 1996; Weissenborn \& Duka, 2003), eyehand coordination (Fillmore \& Vogel-Sprott, 1996; Fillmore, 2003; King \&

\footnotetext{
${ }^{1}$ Address correspondence to Kenta Matsumura, Ph.D., Department of Adult Mental Health, National Institute of Mental Health, National Center of Neurology and Psychiatry, 4-1-1 Ogawa-Higashi, Kodaira, Tokyo, Japan 187-8553 or e-mail (kenta@ncnp.go.jp).

2The authors' sincere thanks to Prof. Peter Rolfe, OBH Ltd., UK, for his assistance in preparing the manuscript.

${ }^{3}$ National Police Agency. (2009, February 26) Motor vehicle accident statistics 2008. Retrieved November 9, 2009 from http://www.e-stat.go.jp/SG1/estat/List.do?lid=000001054077.
} 
Byars, 2004; Brumback, Cao, \& King, 2007), and decision making (George, Rogers, \& Duka, 2005). Of greater importance is that such impairment is more pronounced on more difficult or complex tasks such as those needed in driving than on easier or simpler tasks (Moskowitz, Burns, \& Williams, 1985; Hindmarch, et al., 1991; Hunt \& Witt, 1994).

In attempts to prevent alcohol-impaired driving, alcohol ignition-interlock devices have been developed, mostly based on breath alcohol detection. The advantage of breath-alcohol concentration (BrAC) methods is that they have high correlation with blood alcohol concentration (BAC), thereby meeting the requirements of law enforcement. On the other hand, the assumption that $\mathrm{BrAC}$ alone is an accurate indicator of impairment is not always valid because the relation of BrAC with impairment is modulated by many factors such as age (Linnoila, Erwin, Ramm, \& Cleveland, 1980), acute tolerance (Schweizer \& Vogel-Sprott, 2008), chronic tolerance or drinking habit (Fillmore \& Vogel-Sprott, 1996), task differences (Fogarty \& Vogel-Sprott, 2002), or task difficulty and complexity (Moskowitz, et al., 1985; Hindmarch, et al., 1991; Hunt \& Witt, 1994). Thus, in addition to BrAC-based devices, one may argue that, to improve traffic safety, there is a need for performance-based approaches with which impairment is directly measured using psychomotor or cognitive tests previously shown to be degraded by drinking alcohol.

Attempts to develop such performance-based devices began as early as the 1970s. In this period, the Phystester (Jones \& Tennant, 1972), an alcohol-detecting device using a short-term memory task, and the Critical Tracking Task unit (Thompson, Tennant, \& Repa, 1975), a device based on a psychomotor tracking task, were reported. In the 1980s, the first field test of an on-board alcohol detector using the critical tracking task was conducted and some encouraging results were reported (Allen, Stein, \& Jex, 1984). However, the development of these prototype devices did not continue because they did not meet apparently contradictory technical specifications, such as requiring little time but being accurate and easily implementable, which are necessary requirements for on-board vehicle use (Thompson, et al., 1975). Thus, for any new device to become widely implemented, no matter how promising it may appear, good usability is vital.

Accordingly, this work represents an effort to address these practical problems by introducing four newly developed, rapid performance tests which appear suitable for use with widely available personal computers and mobile phones. These tests have been tentatively named Spiral for iPhone, Spiral for Mac, Modified Mental Rotation, and Catch the Rabbit. The first two are psychomotor tracking tests, whereas the last two are cognitive tests. These were chosen according to the following criteria: (a) 
simple enough to run on mobile phones, (b) possible to complete within 2 min., and (c) different from one another so a wider range of skills fundamental to operating motor vehicles safely could be covered. In addition, they must be (d) designed to be conducted by meeting sequentially organized multiple subgoals both to record a sufficient number of trials within a relatively short time and to heighten the performers' motivation by enabling them to finish tests quickly in accord with their effort.

The first aim of this study was to evaluate the suitability of these four tests for measuring alcohol-induced impairment by comparing scores from participants at a predrink baseline and at three progressive amounts of alcohol intake. The second aim was to evaluate the correspondence, i.e., alternate-form reliability (Parrott, 1991), of the two Spiral tests, which are essentially the same except for the devices which run them.

\section{Method}

\section{Participants}

Eleven men, whose mean age was 23.3 years $(S D=3.6)$, participated. All were recruited from Kanazawa University and came to the laboratory having fasted for at least $12 \mathrm{hr}$. immediately prior to the experiment. None of the participants reported any current or past history of drug or alcohol abuse, although this was not verified by screening tests. Each participant received 3,000 Yen (about U.S. \$30) as a reward after the experiment.

\section{Apparatus}

The performance tests were developed and conducted using a personal computer (Apple, Mac mini, Mac OS X 10.5.4) with a 20-in. display (DELL, 2007WFP HAS), and a mobile phone containing an acceleration sensor (Apple, iPhone 3G, iPhone OS 2.0).

\section{Performance Tests and Test Set}

Four performance tests, Spiral for iPhone, Spiral for Mac, Modified Mental Rotation, and Catch the Rabbit, were used. In developing these tests, much of the software code utilized in previous studies of stressful tasks (Matsumura \& Sawada, 2004, 2009) was included. Participants were required on all tests to maximize both the speed and accuracy of their performance as much as they could.

For the Spiral for iPhone, participants were required to trace a spiralshaped track smoothly from the center segment to the final outermost segment, using a cross-hair cursor on the screen (see Fig. 1). The cursor moves in response to the iPhone's tilt with respect to gravitational force. The segments which constitute components of the spiral-shaped track disappear successively from the screen as the cursor crosses the boundaries between segments, so that the participant can visualize the progress of the task di- 

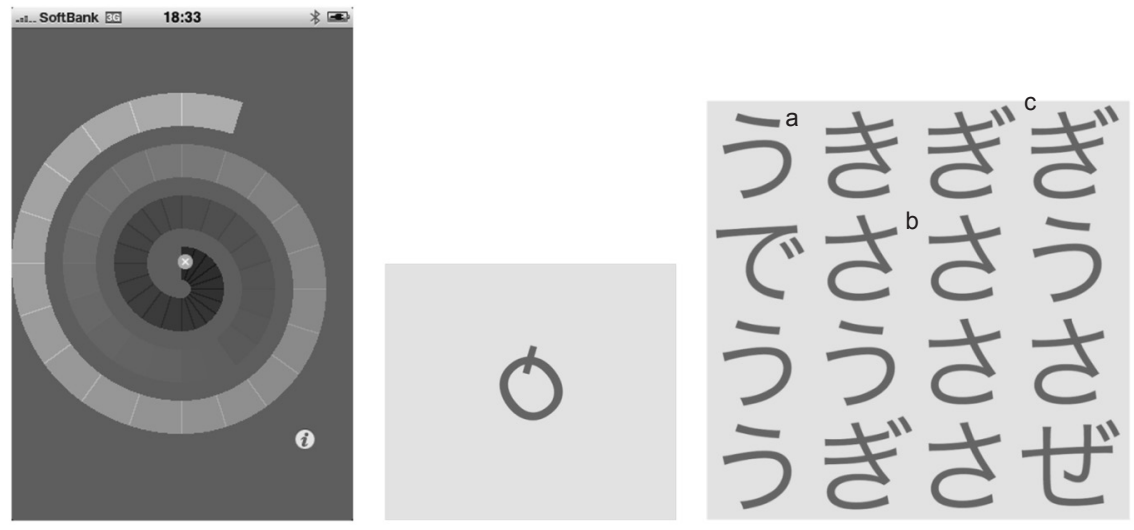

FIg. 1. Examples of ongoing tests on a display. Left: the initial screen of Spiral for the iPhone. Middle: the mirror image Q in Modified Mental Rotation. Right: a $4 \times 4$ hiragana matrix of Catch the Rabbit with descriptive characters. In this case, the target as are A3, B3, and D2. See text for details.

rectly and in a straightforward manner. In case of deviation, the cursor has to be returned to the segment from which it deviated to continue the test. There are three trials, defined as the period from entering the first segment to exiting the last segment.

The Spiral for Mac test is essentially the same as the Spiral for iPhone test described above. The differences are that for the Mac test the cursor is a small square instead of the cross-hair used with the Spiral for iPhone, and a computer mouse is used for the Spiral for Mac instead of the tilt input used with the iPhone.

On the Modified Mental Rotation test, participants were required to answer, by pressing keys, whether a displayed alphabet character was a normal image or a mirror image, regardless of its rotation (see Fig. 1). There are three properties in alphabets: firstly, the character, may be any one of F, G, J, L, P, Q, or R; secondly, the rotation angle may be any one of $-144^{\circ},-108^{\circ},-72^{\circ},-36^{\circ}, 36^{\circ}, 72^{\circ}, 108^{\circ}$, or $144^{\circ}$ angles; thirdly, the image may be either normal or mirror. Thus, this test has $112(=7 \times 8 \times 2)$ trials. The order of each trial was based on a pseudorandom number sequence, the seed of which was derived from the test start time. The intertrial interval was $0.3 \mathrm{sec}$.

For the Catch the Rabbit test, participants were required to indicate when a hiragana Japanese syllabary character, $a$, was followed by $b$ and $c$ buried in a 4 by 4 hiragana matrix, by clicking on $a$ (see Fig. 1). English for $a b c$ is rabbit. There are at least three target $a$ s and at least one distractor $a$ in one matrix. Hidden $a b c s$ are always lined up in alignment, although their 
direction can be in all eight ways; that is, left to right, right to left, top to bottom, bottom to top, top left to bottom right, bottom right to top left, top right to bottom left, or bottom left to top right. Once again, the matrix is created by using a pseudorandom number sequence with a seed derived from the test start-time and selected to meet the above-mentioned criterion. This test has 30 trials. The intertrial interval was $0.3 \mathrm{sec}$.

Below we will refer to the set of 3 Spiral for iPhone trials, 112 Modified Mental Rotation trials, 30 Catch the Rabbit trials, and 3 Spiral for Mac trials as one test set.

Alcohol

The drink used was 96 proof straight vodka. Three percents of target blood alcohol concentration (BACs) were set to $0.02,0.06$, and $0.12 \%$ BAC. These were set to sandwich 0.03 and $0.08 \%$ BAC; that is, the legal driving limits, so-called per se law, in Japan and in the USA, respectively.

The volume to drink was calculated individually by the following formula:

$$
\text { volume }(\mathrm{ml})=833 \times \text { body weight }(\mathrm{kg}) \times \mathrm{BAC}(\%) / 96
$$

where 833 is the constant calculated from specific gravity of alcohol $0.8 \mathrm{~g} /$ $\mathrm{ml}$ and body fluid ratio two-thirds, and 96 is percent proof.

\section{Procedure}

The participant sat in a chair in front of a display in a sound-attenuated, temperature-controlled room. After reading and signing an informed consent form, a catheter was inserted into the brachial artery of the dominant hand. The handedness was determined using a Japanese adapted version (Negishi, Maehara, \& Momose, 1990; Soshi \& Hagiwara, 2004) of the Edinburgh Inventory (Oldfield, 1971). They were given instructions regarding the test set and were then allowed to practice one complete set.

During the experiment, participants drank vodka three times, 10 to $15 \mathrm{~min}$., 40 to $45 \mathrm{~min}$., and 70 to $75 \mathrm{~min}$. after the start, the amounts equivalent to $0.02,0.06$, and $0.12 \%$ BAC, respectively. The drawing of blood and the completion of test sets were conducted four times; after $0(0-\mathrm{min}$. condition), 35 (35-min. condition), 65 (65-min. condition), and 95 (95-min. condition) minutes from the beginning of the experiment.

After BrAC had fallen below $0.15 \%$, which corresponds to $0.03 \% \mathrm{BAC}$, participants were allowed to leave the laboratory.

Performance Measures and Data Analyses

For the Spiral for iPhone and the Spiral for Mac tests, the periods on the track and out of the track, and the number of deviations from the track were measured in a trial. For the Modified Mental Rotation and the Catch 
the Rabbit tests, reaction time in sec. and whether answers were correct or incorrect were measured for a trial. Then the mean reaction time of correct trials and the mean rate of correct answers were calculated.

The trials were divided into three, and performance measures for each third were calculated; then arcsine and square root transformations were applied to the values for rate and time, respectively. For the 0-min. condition, following Fillmore and Vogel-Sprott's method (1996), considering that there is a trial-and-error learning effect, the best of the three was used as the baseline approximate performance. For the 35-, 65-, and 95min. conditions, the mean of the three conditions was used as the performance index.

The effect of each condition was analyzed by one-way analysis of variance, using the randomized blocks method and, where appropriate, Tukey's Honestly Significant Difference tests were conducted.

\section{Results}

For the 0-min. and 35-min. conditions, all participants completed the test sets. For the 65-min. condition, one participant was intoxicated and could not complete the Spiral for Mac. For the 95-min. condition, three participants, including the above-mentioned one, were intoxicated and could not complete the subtasks set.

Mean (SEM) values of BACs, performance measures of Spiral for iPhone, Modified Mental Rotation, Catch the Rabbit, and Spiral for Mac as a function of condition are presented in Table 1 . The results of statistical analyses, $F$ ratios, values of partial $\eta^{2}$, and follow-up tests, are also presented in Table 1.

Predicted reliability $\rho$ for Spirals was calculated using the SpearmanBrown prophecy formula. $\rho$ values for the durations of being on track and out of track with the number of deviations were $.49, .83$, and .86 , respectively.

\section{Discussion}

The first aim was to evaluate the potential of four newly developed, rapidly administered, computerized, easily implementable performance tests, named Spiral for iPhone, Modified Mental Rotation, Catch the Rabbit, and Spiral for Mac, for measuring alcohol-induced impairment. To attain this, the performance of participants at $0.000 \%$ BAC was compared with their performance during three progressive increased BAC levels. Analyses showed decreases in performance accuracy on all tests at $0.111 \%$ BAC, and also in all except Spiral for iPhone at $0.062 \%$ BAC. Clearly, these results suggest all tests measure alcohol-induced impairment.

Decreases in performance were apparent in accuracy-related measures. Taken together with the inconsistent findings that speed after drink- 
TABLE 1

Means and SEMs For Various Indices by Condition and Results of Statistical Analyses

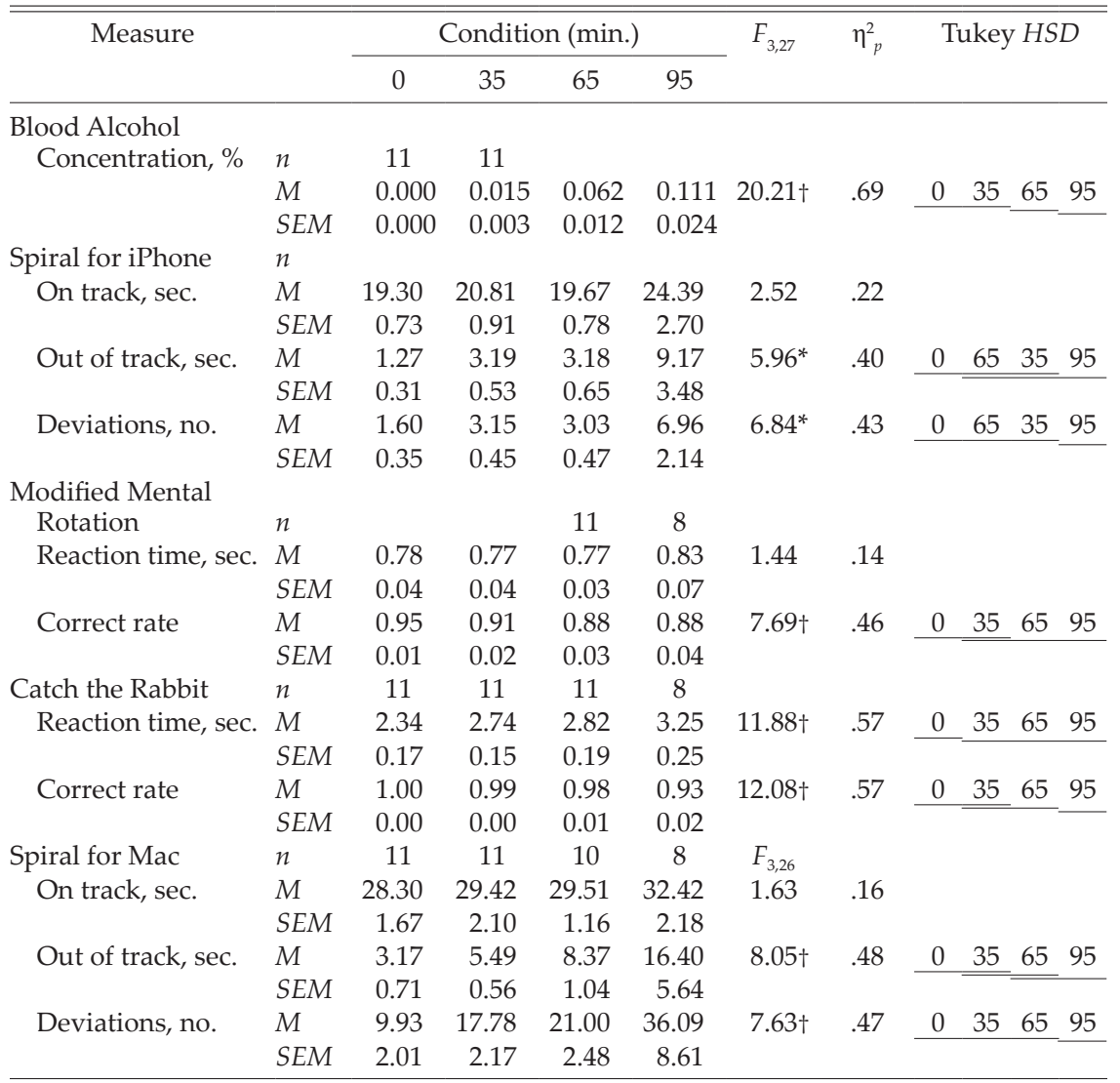

$\overline{\text { Note. }- \text { Conditions underlined together are not significantly different from each other. } \dagger p<}$ $.001 .{ }^{*} p<.01$.

ing can be both faster (e.g., Zhu, Volkow, Ma, Fowler, \& Wang, 2004; Rose \& Duka, 2007) and slower (e.g., Gustafson \& Källmén, 1990), one might believe that the only tests needed are accuracy-related measures. However, the relation between speed and accuracy can vary according to participants' strategy. For example, it has been shown that participants can compensate for alcohol-impaired accuracy by slowing their performance intentionally (Gustafson \& Källmén, 1990). Thus, in performance-based devices, impairment of both accuracy and speed should be evaluated.

In this study, only the single influence of alcohol was examined. Thus, one does not know the specificity of these tests for alcohol-induced impairment. However, even if one considers these tests to have low specificity, i.e., low discriminant validity, this may not actually be problemat- 
ic. On the contrary, the tests might well have an advantage over the use of breath alcohol concentration measures. This is because such generality permits detection of impairment caused by nonspecific factors such as drugs (Hindmarch, et al., 1991), fatigue, poor physical condition, and so on (Thompson, et al., 1975). This contrasts with breath alcohol concentration-based devices which are highly specific for alcohol. It should not be forgotten that the ultimate concern is impairment, rather than the cause.

Values of alternate-form reliability (Parrott, 1991) for two of the psychomotor tests evaluated, based on Spirals, were high on the accuracy-related measures, suggesting that the Spiral test is not susceptible to differences in the devices on which the Spirals tests are run, i.e., one is a desktop computer with mouse input and large screen, the other is a handheld mobile phone with tilt input and a small touch screen. Considering that psychomotor tests are by their nature more vulnerable to such differences than cognitive tests, it seems highly likely that Modified Mental Rotation and Catch the Rabbit are scarcely affected by these differences. This notion is encouraging of the development of the Modified Mental Rotation test for iPhone and the Catch the Rabbit for iPhone as convenient alternatives.

The experimental design in this study has some limitations, in that, firstly, the alcohol-intake treatments were not randomized, secondly, there was no placebo group, and, thirdly, the sample size and range were small and narrow, respectively. Thus, possible confounders, such as learning effects, could not be eliminated. However, with this experimental design, learning effects should only occur at progressively increased amounts of alcohol and so should not falsely strengthen the data. Further, although the participant population was not fully representative of motor vehicle operators, it was representative of those having higher crash rates ${ }^{3}$, and so was a suitable population for the present tests. In subsequent studies, usefulness of these tests for measuring alcohol-induced impairment should be evaluated with a wider range of participants, including experts on these tests, and using more sophisticated experimental designs.

Electronic technologies, including new families of communication and computing devices, continue to evolve rapidly and enter common use. This study of computerized tests might be a first step in facilitating their use as a practical ignition-interlock system in motor vehicles.

\section{REFERENCES}

Allen, R. W., Stein, A. C., \& Jex, H. R. (1984) Field test of a drunk driving warning system (DDWS). In American Association for Automotive Medicine, 28th Annual Proceedings. October 8-10, Denver, Colorado. Pp. 261-272.

Brumback, T., CaO, D., \& KING, A. (2007) Effects of alcohol on psychomotor performance and perceived impairment in heavy binge social drinkers. Drug and Alcohol Dependence, 91, 10-17. 
Fillmore, M. T. (2003) Reliability of a computerized assessment of psychomotor performance and its sensitivity to alcohol-induced impairment. Perceptual and Motor Skills, 97, 21-34.

Fillmore, M. T., \& Vogel-Sprott, M. (1996) Social drinking history, behavioral tolerance and the expectation of alcohol. Psychopharmacology, 127, 359-364.

Fogarty, J. N., \& Vogel-Sprott, M. (2002) Cognitive processes and motor skills differ in sensitivity to alcohol impairment. Journal of Studies on Alcohol, 63, 404-411.

George, S., Rogers, R. D., \& Duka, T. (2005) The acute effect of alcohol on decision making in social drinkers. Psychopharmacology, 182, 160-169.

Gustafson, R. (1986) Alcohol and vigilance performance: effect of small doses of alcohol on simple visual reaction time. Perceptual and Motor Skills, 62, 951-955.

Gustafson, R., \& KällméN, H. (1990) Alcohol and the compensation hypothesis: a test with cognitive and psychomotor tasks. Perceptual and Motor Skills, 71, 1367-1374.

Hindmarch, I., Kerr, J. S., \& Sherwood, N. (1991) The effect of alcohol and other drugs on psychomotor performance and cognitive function. Alcohol and Alcoholism, 26, 71-79.

Hunt, W. A., \& Witt, E. D. (1994) Behavioral effects of alcohol ingestion: implications for drug testing. Toxic Substances Journal, 13, 41-49.

Jones, T. O., \& Tennant, J. A. (1972) A critical evaluation of the Phystester: a test for driver impairment. American Association for Automotive Medicine, 16th Annual Proceedings. October 19-21, Chapel Hill, North Carolina. Pp. 274-306.

KING, A. C., \& Byars, J. A. (2004) Alcohol-induced performance impairment in heavy episodic and light social drinkers. Journal of Studies on Alcohol and Drugs, 65, 27-36.

Koelega, H. S. (1995) Alcohol and vigilance performance: a review. Psychopharmacology, 118, 233-249.

Linnoila, M., Erwin, C. W., Ramm, D., \& Cleveland, W. P. (1980) Effects of age and alcohol on psychomotor performance of men. Journal of Studies on Alcohol and Drugs, 41, 488-495.

MAtsumura, K., \& SAWADA, Y. (2004) [Controllability and hemodynamic reaction patterns during mental stress]. [Japanese Journal of Physiological Psychology and Psychophysiology], 22, 247-255. [in Japanese]

Matsumura, K., \& Sawada, Y. (2009) [Cardiovascular responses during two kinds of mental arithmetic tasks]. [The Japanese Journal of Psychology], 79, 473-480. [in Japanese]

Matthews, D. B., Best, P. J., White, A. M., Vandergriff, J. L., \& Simson, P. E. (1996) Ethanol impairs spatial cognitive processing: new behavioral and electrophysiological findings. Current Directions in Psychological Science, 5, 111-115.

Mitchell, M. C. (1985) Alcohol-induced impairment of central nervous system function: behavioral skills involved in driving. Journal of Studies on Alcohol: Supplement, 10, 109-116.

Mosкowitz, H., \& Burns, M. (1990) Effects of alcohol on driving performance. Alcohol Health \& Research World, 14, 12-14.

Moskowitz, H., Burns, M. M., \& Williams, A. F. (1985) Skills performance at low blood alcohol levels. Journal of Studies on Alcohol, 46, 482-485.

Negishi, N., Maehara, K., \& Momose, T. (1990) [Handedness and Criminals]. [Correctional Medicine], 39, 40-47. [in Japanese] 
Oldfield, R. C. (1971) The assessment and analysis of handedness: the Edinburgh Inventory. Neuropsychologia, 9, 97-113.

Parrotr, A. C. (1991) Performance tests in human psychopharmacology(1): test reliability and standardization. Human Psychopharmacology, 6, 1-9.

Rose, A. K., \& Duka, T. (2007) The influence of alcohol on basic motoric and cognitive disinhibition. Alcohol and Alcoholism, 42, 544-551.

Schweizer, T. A., \& Vogel-Sprott, M. (2008) Alcohol-impaired speed and accuracy of cognitive functions: a review of acute tolerance and recovery of cognitive performance. Experimental and Clinical Psychopharmacology, 16, 240-250.

Soshi, T., \& Hagiwara, H. (2004) Asymmetry in linguistic dependency: linguistic and psychological studies of Japanese right dislocation. English Linguistics, 21, 409-453.

Stapleton, J. M., Guthrie, S., \& Linnoila, M. (1986) Effects of alcohol and other psychotropic drugs on eye movements: relevance to traffic safety. Journal of Studies on Alcohol, 47, 426-432.

Thompson, R. R., Tennant, J. A., \& Repa, B. S. (1975) Vehicle-borne drunk driver countermeasures. In S. Israelstam \& S. Lambert (Eds.), Proceedings of the 6 th International Conference on Alcohol, Drugs and Traffic Safety. September 8-11, Toronto, Ontario, Canada. Pp. 347-363.

Weissenborn, R., \& DukA, T. (2003) Acute alcohol effects on cognitive function in social drinkers: their relationship to drinking habits. Psychopharmacology, 165, 306312.

Zhu, W., Volkow, N. D., MA, Y., Fowler, J. S., \& WANG, G. J. (2004) Relationship between ethanol-induced changes in brain regional metabolism and its motor, behavioural and cognitive effects. Alcohol and Alcoholism, 39, 53-58.

Accepted November 13, 2009. 